\title{
THE RANKINE GAS FLOW IN THE HODOGRAPH PLANE*
}

\section{BY MAX M. MUNK (White Oak, Maryland)}

Introduction. In the theory of plane two-dimensional potential flows of a perfect incompressible fluid, the flow associated with the superposition of one point source and of the parallel flow is known as the Rankine flow. When transforming into hodograph variables and when choosing the Legendre reciprocal potential $L$ as the dependent variable, the Rankine flow is represented by a sole point source positioned at a velocity point representing the velocity at infinity in the physical plane.

It is the aim of this paper to set up a corresponding hodograph solution for a perfect gas, arriving with subsonic velocity and flowing past the head of an obstacle extending infinitely far downstream. We shall have to employ the stream function $\psi$ in the physical plane which is associated with the physical potential $\phi$, and we shall have to employ and will primarily deal with the Legendre reciprocal potential $L$ in the hodograph plane, which is associated with a reciprocal stream function $\xi$. The functions $\phi$ and $\xi$ will not be used in the numerical computations.

We recall that the sets of differential equations for these quantities in terms of the hodograph variables $w$ and $\theta$ are the following ones, with $Q$ being written for

$$
Q=\left(1-\frac{\gamma+1}{\gamma-1} w^{2}\right) /\left(1-w^{2}\right)
$$

by way of abbreviation:

$$
\begin{aligned}
\frac{1}{w} \frac{\partial \xi}{\partial \theta} & =\rho \frac{\partial L}{\partial w}, \\
\frac{\partial \xi}{\partial w} & =-\frac{Q \rho}{w} \frac{\partial L}{\partial \theta}, \\
\frac{1}{w} \frac{\partial \phi}{\partial \theta} & =\frac{1}{\rho} \frac{\partial \psi}{\partial w}, \\
\frac{\partial \phi}{\partial w} & =-\frac{Q}{\rho} \frac{\partial \psi}{\partial \theta} .
\end{aligned}
$$

The velocity $w$ is expressed in units consistent with the following. The velocity inclination angle is denoted by $\theta$ (its square is often denoted by $\tau$ ), $\rho$ denotes the density $\rho=\left(1-w^{2}\right)^{1 /(\gamma-1)}$ and $\gamma$ denotes the expansion exponent of the gas. The Legendre transformation employed relates to the Cartesian coordinates in the physical plane and in the hodograph. It does not relate to polar coordinates.

Equations (1) and (2) possess particular solutions of the form

$$
\begin{aligned}
L(w, \theta) & =L_{n}(w) \exp i n \theta, \\
\xi(w, \theta) & =\xi_{n}(w) \exp i(n \theta+\pi / 2), \\
\psi(w, \theta) & =\psi_{n}(w) \exp i(n \theta-\pi / 2),
\end{aligned}
$$

*Received Apr. 19, 1950. This work was sponsored by the Office of Naval Research. 
$L_{n}$ being a solution of

$$
w \frac{d}{d w}\left[\rho w \frac{d L_{n}}{d w}\right]-n^{2} \rho Q L_{n}=0
$$

In this paper, only integer values of the parameter $n$ will occur. The special solution of (1a) which becomes zero at $w=0$ will be designated as $L_{n}$. Replacing in such a solution the integer $n$ by $-n$ does not lead to a new independent solution. The symbol $L_{-n}$ will be used in this paper to denote a second solution of (1a) which is independent of the first solution $L_{n}$ but which is not its analytic continuation with reference to $n$, and which is not obtained by substituting $-n$ for $+n$. In this paper, $n$ is always positive.

Substituting for the subsonic velocity $w$ a "distorted" velocity $s$ defined by

$$
d s / s=\sqrt{Q} d w / w,
$$

We transform the two sets (1) and (2) into similar sets (1b) and (2b) superior to (1a) and (2a) in symmetry, in that then in each pair of equations the same factor appears on the right hand side:

$$
\begin{aligned}
\frac{1}{s} \frac{\partial \xi}{\partial \theta} & =\rho \sqrt{Q} \frac{\partial L}{\partial s}, \\
\frac{\partial \xi}{\partial s} & =-\frac{\rho \sqrt{Q}}{s} \frac{\partial L}{\partial \theta}, \\
\frac{1}{s} \frac{\partial \phi}{\partial \theta} & =\frac{1}{\rho} \sqrt{Q} \frac{\partial \psi}{\partial s}, \\
\frac{\partial \phi}{\partial s} & =-\frac{\sqrt{Q}}{\rho s} \frac{\partial \psi}{\partial \theta} .
\end{aligned}
$$

Equations (1b) and (2b) can be interpreted as relating to the steady flow say of electricity or of heat in a disc occupying the distorted hodograph plane. The disc has variable conductivity (or resistance) the conductivity being a function of the distance from the center but not of the flow direction. This conductivity is proportional to $\rho \sqrt{Q}$ for (1b) or to $\sqrt{Q} / \rho$ for (2b), and becomes zero at the sonic speed $Q=0$.

This indicates that for small variations of $s$, the solutions in the distorted subsonic portion of the hodograph must approach solutions of Laplace's equation. If the resistance changes are very gradual compared with the changes of the potential, an approximate solution can be obtained from a solution of Laplace's equation by increasing the potential gradients and decreasing the stream densities in the same ratio given by the square root of the conductivity. This thought leads to the so-called asymptotic particular solutions of (1) and (2) which are approached by the exact solutions as $n$ approaches infinity. The asymptotic solutions are

$$
L=\rho^{-1 / 2} Q^{-1 / 4} S^{ \pm n} \exp i n \theta
$$

and similarly for the stream function. In the supersonic regime an analogous relation holds, the wave equation replacing Laplace's equation, and the sine or cosine of the logarithm of $s$ replacing the power of $s$. These asymptotic relations break down at the sonic speed. They can be obtained or verified by inserting the multiplier of the exponential 
function in (5) into (1a), and setting the terms containing the two highest powers of $n$ equal to zero. This gives two ordinary differential equations for $s^{n}$ and its factor.

The asymptotic behaviour of the reciprocal potential $L$ and of its associated stream function $\psi$ clarifies the character of the infinite series in terms of $L_{n}$ or $\psi_{n}$ to be established. Their convergence and the type of singularity represented is determined by the terms of higher order and just these terms are described by the asymptotic expressions. The series are thus recognized to behave in many respects like the corresponding series relating to Laplace's equation.

Sets (1) and (2) are closely related to each other and in consequence the functions $L_{n}$ and $\psi_{n}$ are also closely related. The following relations hold for all particular solutions, whether designated by $n$ or by $-n(n>1)$ :

$$
\begin{gathered}
\psi_{n}=\rho\left[n L_{n}-\frac{w}{n} \frac{\partial L_{n}}{\partial w}\right], \\
L_{n}=\frac{1}{\rho\left(n^{2}-1\right)}\left[n \psi_{n}+\frac{w}{n} \frac{\partial \psi_{n}}{\partial w}\right], \\
\frac{\partial\left(\psi_{n} w^{ \pm n}\right)}{\partial w}=\rho( \pm n-1) \frac{\partial\left(L_{n} \frac{\left.w^{ \pm n}\right)}{\partial n} .\right.}{}
\end{gathered}
$$

Note that for the first solution the stream function multiplier associated with the reciprocal potential is

$$
\psi_{n}=\left[\frac{L_{n}}{w^{n}}\right]_{w=0}(n-1) w^{n} F\left(a, b ; c ; w^{2}\right)
$$

where the hypergeometric series is set up with parameters $a, b$, and $c$ which are appropriate for the stream function and for the value of $n$.

In (6) to (8) $n$ has to be taken positive if so taken in (3).

The Rankine flow. In the theory of the potential flows of the ideal incompressible fluid we are attracted by the Rankine flow on account of its simplicity from the mathematical view point. In the complex plane $z=x+i y$ representing the physical plane the potential is the real part of a complex variable $P$

$$
\begin{aligned}
& P=\log z+z \\
& \zeta=d P / d z \\
& L=-P+z \zeta=\log (1-\zeta)+1
\end{aligned}
$$

Equations (10) presume unit velocity at infinity and unit source velocity at unit distance from the source. The source is at the origin of the coordinate system $x, y$. The impact point is at $x=-1$, the flow is in the $x$ direction. The vertical $x=-\frac{1}{2}$ is the line of constant velocity magnitude equal to the velocity magnitude at infinity. In the hodograph this line is transformed into the circle $w=1$, containing the hodograph source. This circle will be called the singularity circle.

We have to elect the features of the flow of the incompressible fluid which we wish it to share with the corresponding gas flow. We are guided in this choice by expediency 
with regard to the mathematical treatment. We emphasize the condition at infinity. We not only require there the velocity to be constant and parallel. We furthermore require that the conditions at infinity be represented in the hodograph plane by a single source. We find it finally desirable to derive the series for $L$ in terms of $L_{n}$ directly from the corresponding series for the incompressible fluid. It is therefore desirable to require that at the singularity circle a suitable series should agree term by term with the corresponding series for the incompressible fluid. A quantity acceptable for that is $\partial L / \partial w$. This is equivalent to the requirement that the lines connecting pairs of points of equal velocity-direction and of undisturbed velocity magnitude relating to the incompressible fluid and to the gas be normal to the velocity in question. This implies that the curve of undisturbed velocity magnitude for the gas have a vertical asymptote $x=$ const.

The solution for $L$ is given by two series applicable respectively to the inside and the outside of the singularity circle. They are determined (a) by the condition that each term be a solution of (1), (b) that the differential quotient with respect to $w$ of each term be equal to the corresponding quantity applying to the incompressible flow, and (c) that the terms of the two series approach the same value at the singularity circle except at the singularity point itself. Let the "prime" denote differentiation with respect to $w$. Then for the incompressible flow we have

$$
\begin{gathered}
S_{1}=-\sum_{n=1}^{\infty}(1 / n) w^{n} \cos n \theta \\
S_{1}^{\prime}=-\sum_{n=1}^{\infty} w^{n-1} \cos n \theta \\
S_{2}=\log w-\sum_{n=1}^{\infty}(1 / n) w^{-n} \cos n \theta \\
S_{2}^{\prime}=\sum_{n=0}^{\infty} w^{-n-1} \cos n \theta
\end{gathered}
$$

The corresponding series for the gas flow are

$$
\begin{gathered}
S_{1}=-\sum_{n=1}^{\infty} \frac{L_{n} \cos n \theta}{\left[L_{n}^{\prime}\right]_{a}} \\
S_{2}=-\sum_{n=1}^{\infty}\left[\frac{L_{n} L_{-n}^{\prime}+L_{-n} L_{n}^{\prime}}{L_{n} L_{-n}^{\prime}-L_{-n} L_{n}^{\prime}} \cdot \frac{1}{L_{n}^{\prime}}\right]_{c} L_{n} \cos n \theta \\
+\sum_{n=0}^{\infty}\left[\frac{2 L_{n}}{L_{n} L_{-n}^{\prime}-L_{-n} L_{n}^{\prime}}\right]_{s} L_{-n} \cos n \theta
\end{gathered}
$$

The subscript $s$ denotes the value of the quantity at the singularity circle, which latter is subsonic.

Concluding remarks. The position in the physical plane associated with any hodograph point is equal to the gradient of $L$. The stream function $\psi$ is computed by applying (6) and (9) to the solutions (13) and (14) term by term.

Modifications of the solution can be obtained by adding through the entire hodograph plane a finite number of terms containing the $L_{n}$ ( $n$ positive) or even an infinite series 
of such terms converging appropriately. The method can also be applied to other singularities of integer order.

APPENDIX. On the choice of the second solution if $n$ is integer.

The differential equation for $L_{n}$ is related to the hypergeometric equation. It imposes a definite ratio on pairs of consecutive terms of a power series representing a solution. This ratio depends on the coefficients of the differential equation and on the order or power of the two terms. It is therefore a straight-forward procedure to write down a series $R_{n}$ extending infinitely to the ascending and to the descending side complying with this requirement, starting with a term $A x^{\sigma}$ where $A$ and $\sigma$ are arbitrarily chosen. Any power series actually representing a solution must be a special case of $R_{n}$, namely $\sigma$ must have the proper value. This special value is computed from the so-called indicial equation, obtained by setting the multiplier of any one term of $R_{n}$, preferably the term of power $\sigma-1$ equal to zero. The series breaks then off on one side, in this case on the descending side, and the terms in ascending powers may form a convergent series. The indicial equation is of second degree and ordinarily furnishes two roots, namely $\sigma=n$ and $\sigma=-n$ in the present case.

Differentiating $R_{n}$ with respect to $\sigma$ leads to a second series (not a power series) which under proper circumstances may also represent a solution.

If $n$ is an integer, the two series derived from $R_{n}$ have terms of equal power in common, and therefore at best would represent substantially the same solution. In this case, the series $\partial R_{n} / \partial \sigma$ furnishes a second solution independent of the first solution. But any product $R_{n}$ multiplied by any function of $\sigma$ would likewise, and when differentiated would not furnish the same second solution. The second solution is general, in that it contains two integration constants.

No substantial reasons have become known to this writer why to select one particular second solution in preference to another. The solution ordinarily considered as standard is derived from $R_{n}$ in the manner described and not from $R_{n}$ multiplied by a function of $\sigma$. In the series representation of that solution, the hypergeometric series appears multiplied by $\log \left(A x^{p}\right)$, where $A=1$.

Note on the convergence of (13) and (14). By A. Van Tuyl. These series are convergent as a direct consequence of the asymptotic expressions for $L_{\star_{n}}$ and $L_{*_{n}}^{\prime}$ implied in (5). The proof of these asymptotic relations follows from a more general result of J. Horn [4] from which we have

$$
\begin{aligned}
& L_{ \pm_{n}}=\rho^{-1 / 2} Q^{-1 / 4} s^{ \pm n}\left[1+O\left(\frac{1}{n}\right)\right], \\
& L_{ \pm_{n}}^{\prime}= \pm n s^{\prime} \rho^{-1 / 2} Q^{-1 / 4} s^{ \pm n-1}\left[1+O\left(\frac{1}{n}\right)\right] .
\end{aligned}
$$

Denoting the value of $s$ at the singularity circle by $s_{0}$, we find

$$
\begin{gathered}
\frac{L_{n}}{\left[L_{n}^{\prime}\right]_{s}}=\left(\frac{s}{s_{0}}\right)^{n} \cdot O\left(\frac{1}{n}\right), \quad\left[\frac{L_{n} L_{-n}^{\prime}+}{L_{n} L_{-n}^{\prime}-} \frac{L_{-n} L_{n}^{\prime}}{L_{-n} L_{n}^{\prime}} \cdot \frac{1}{L_{n}^{\prime}}\right]_{s} L_{n}=\left(\frac{s_{0}}{s}\right)^{n} O\left(\frac{1}{n^{2}}\right), \\
{\left[\frac{2 L_{n}}{L_{n} L_{-n}^{\prime}-L_{-n} L_{n}}\right]_{0} L_{-n}^{\prime}=\left(\frac{s_{0}}{s}\right)^{n} O\left(\frac{1}{n}\right) .}
\end{gathered}
$$


From this follows the convergence of (13) for $s<s_{0}$, the interior of the singularity circle, and the convergence of (14) for $s>s_{0}$. The reader may wish to compare this with reference [1], pp. 46-49.

\title{
REFERENCES
}

1. Chaplygin, Gas jets, Moscow 1902, see also NACA TM 1063.

2. Goldstein, Lighthill and Craggs, On the hodrograph transformation for highspeed flow, Q. J. of Mech. Appl. Math. 1, 344-357 (1948).

3. Garrick and Kaplan, On the flow of a compressible fluid by the hodograph method, NACA Report No. 790 (1944).

4. J. Horn, Ueber eine lineare Differentialgleichung zweiter Ordnung mit einem willkuerlichen Parameter, Math. Ann. 52, 271-292 (1899).

\section{ON THE METHOD OF INVERSION IN THE TWO-DIMENSIONAL THEORY OF ELASTICITY*}

\author{
By E. STERNBERG AND R. A. EUBANKS (Illinois Institute of Technology)
}

1. Introduction. The method of inversion, originally introduced by J. H. Michell [1], has led to a variety of technically significant solutions to "plane" problems in the theory of elasticity [2], [3], [4], [5]. The usefulness of Michell's stress-field transformation stems from its invariant properties which assure the preservation of an important class of boundary conditions. In the present note we show that any conformal stress-field transformation which preserves the principal-stress trajectories for every choice of the antecedent Airy function, is essentially a Michell transformation.

2. The Michell transformation. The inversion theorem of Michell may be stated as follows. Let $U(z, \bar{z})$ be real and biharmonic in a region $R$ of the $z$-plane, and let $R^{*}$ be the image of $R$ with respect to the mapping ${ }^{1}$

$$
\zeta=w(z)=\frac{a z+b}{c z+d}, \quad\left|\begin{array}{ll}
a & b \\
c & d
\end{array}\right|=1 .
$$

Then the function

$$
U^{*}(\zeta, \bar{\zeta})=h U[g(\zeta), \overline{g(\zeta)}]
$$

where $h^{2}=\left|w^{\prime}\right|^{2}$ and $g$ is the inverse of $w$, is biharmonic in $R^{*}$. The stress fields generated by $U$ and $U^{*}$, considered as Airy functions in $R$ and $R^{*}$ respectively, are related according to ${ }^{2}$

$$
\left.\begin{array}{c}
\sigma^{*}+i \tau^{*}=\lambda(\sigma+i \tau)+p, \\
\lambda=\frac{1}{h}, \quad p=2\left(\lambda_{z \bar{z}} U-\lambda_{\bar{z}} U_{z}-\lambda_{z} U_{\bar{z}}\right) .
\end{array}\right\}
$$

*Received Mar. 15, 1950.

${ }^{1}$ Michell actually used $\zeta=1 / z$.

${ }^{2}$ Subscripts attached to functions which originally bear no subscripts denote partial differentiation. 